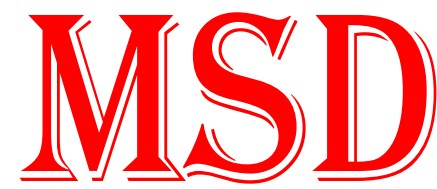

Medical Science and Discovery ISSN: 2148-6832

\title{
Effect of intermittent hypoxic intervention on aerobic and anaerobic performance of the elite athletes
}

\author{
Ali Eroglu ${ }^{1}$, Taner Aydın ${ }^{2}$ \\ 1 Erenköy Physical Medicine and Rehabilitation Hospital, Dept of Sports Medicine, Istanbul, TR \\ 2 Efeler Private Sports Medicine Clinic, Istanbul, TR \\ * Corresponding Author: Ali Eroglu E-mail: dralieroglu@ hotmail.com
}

\section{ABSTRACT}

Objective: The use of hypoxic training has increased to improve the performance of endurance athletes in recent years. Due to not having the suitable conditions and environment for each athlete and team, intermittent hypoxic training has been noted. The purpose of this study is to investigate the effect of intermittent hypoxic training on aerobic and anaerobic performance of elite athletes.

Materials and Methods: A total of 40 elite distance athletes were taken into our study and divided into two groups as hypoxia and normoxia. While using the intermittent intervention for the hypoxic group 5 minutes intervals for a total of 1 hour per day, 3 days per week for a-4 week period, the same normoxic training protocol was used for the normoxic group. Aerobic and anaerobic performance parameters were measured with venous blood samples of the athletes in the first three days before and after hypoxic intervention.

Results: When the hypoxia and normoxia groups were evaluated before and after intermittent hypoxia, there was no statistically change in aerobic and anaerobic performance values $(\mathrm{p}>0.05)$.

Conclusion: We observed that there was not a statistical change of intermittent hypoxic intervention for the performances of hypoxic group. However, the more dose and the duration of hypoxic training, the more amount of performance gain can be achieved.

Keywords: Intermittent hypoxic intervention- aerobic performance-anaerobic performance

\section{INTRODUCTION}

The use of hypoxia training has been increasing in recent years among athletes to increase performance. However, since not every athlete or team has the appropriate geographical environment and conditions for hypoxia training, the use of various hypoxic training methods has been considered. While there is a significant amount of studies investigating the effects of adaptation and response mechanisms that depending on due to the acute and chronic hypoxia on sportive performance, studies on the effects of intermittent hypoxic training are limited (1-4). In general, authors using hypoxic training modalities evaluate that responses to acute or chronic hypoxia differ individually (6-8). The purpose of hypoxic training methods is to increase the performance of the hypoxic adaptation gained (5-8). Due to the difficulties of hypoxic training due to high altitude (such as suitable equipment, cost, and time constraints), various alternative methods and equipment have been developed to gain similar adaptations. While designing hypoxic training equipment and protocols, it is taken into consideration to provide the athlete with the least possible discomfort and maximum performance increase (10). Although the method and protocol to be used for Intermittent Hypoxic Training (IHT) are important, the type of athlete to be used and the dose and duration of hypoxia exposed are also important (9). In the studies of Bonetti and Hopkins, it was evaluated that intermittent hypoxic training could be effective in sub-elite or recreational athletes rather than elite athletes (9). In our study, it was aimed to investigate the effects of intermittent hypoxia application on aerobic and anaerobic performance of elite athletes. 


\section{MATERIAL and METHODS}

\section{Participants}

A total of 40 athletes, 20 from the Gendarmerie Athletic team and 20 elite male long-distance runners from the Turkish Athletics Federation were included in our study.

Twenty of the athletes were included in the hypoxia group, while the other 20 were included as the normoxia group. It was learned from the participants that similar training programs were used before and after the competitions. The height and weight of the athletes were measured with Tanita bc 418 body analysis machine. Following the detailed anamnesis, musculoskeletal examination, ECG, and routine laboratory tests of the athletes recorded, patients with acute respiratory tract infection, a chronic febrile illness, a family history of sudden death, and a chronic sports injury were not included in the study. All athletes volunteered to take part in this study. Written informed consent form was obtained from each participant, and ethical approval was obtained from the Human Research Ethics Committee of the Institution (15/02/11-168).

\section{Intermittent Hypoxic Intervention}

In the study, athletes were divided into two groups as hypoxia and normoxia. The intermittent hypoxic intervention(IHI) procedure used by Hamlin MJ et al. was adapted to our study (18). Hypoxia was applied to the hypoxia group for four weeks, three days a week, for 60 minutes a day, for a total of 6 hours. Five minutes of normal ambient air (normoxia) followed by five minutes of hypoxia $\left(15 \% \mathrm{FiO}_{2}\right)$ were applied during each 60-minute hypoxic condition. The amount of hypoxia was applied by starting with $15 \% \quad \mathrm{FiO} 2$ and decreasing it by $1 \%$ every week to $12 \% \mathrm{FiO}_{2}$ oxygen concentration and $90-95 \% \mathrm{O}_{2}$ saturation during the 4th week. It was observed that there was a correlation between $\mathrm{FiO}_{2}$ application and weekly $\mathrm{O}_{2}$ saturation.

Normoxic group was ventilated with ambient air $\left(21 \% \mathrm{FiO}_{2}\right)$ at five-minute intervals. Subjects were instructed to remove their masks during the normoxia period following each hypoxia period. During the hypoxic intervention, the arterial oxygen saturation and heart rate variability of the individuals were monitored by a portable pulse oximeter from the fingertips. The hypoxic environment was created with the $\mathrm{GO}_{2}$ Altitude hypoxicator (Biomedtech, Victoria, Australia) device that can mimic the oxygen density in the range of 9$21 \%$ with a mouth nose mask. The most important advantage of the $\mathrm{GO}_{2}$ altitude hypoxicator device used in the study is that diluting the oxygen gas in the normal air, providing realtime monitoring of the hypoxic environment within very narrow limits during the application session, as well as an automatic biofeedback system that provides the status of hypoxia. Individuals who could not attend more than two of the 12 total sessions throughout the study were excluded from the study. At the beginning of the study, both groups were told not to train for 1 hour before and after the session.

Before the aerobic and anaerobic performance tests, all the athletes were informed about the study protocol. For the $\mathrm{VO}_{2}$ aerobic performance test, the bicycle ergometer that the athletes would sit on, was specially adjusted for the athlete. ECG electrodes were placed in the appropriate chest leads.
Then, the mouth and face mask were settled on the athlete's head in such a way that they could not get air. During the test, the blood pressure monitor, which was connected to the system, was attached to the right arm and blood pressure was monitored on the monitor along with the pulse (Figure-1) Before the aerobic performance test, the athletes were adapted and warmed up for 2 minutes at a workload of 50 watts with $50-60 \mathrm{rpm}$. In order for the athletes to reach the maximum exercise intensity, the system was automatically increased by 25 watts every 3 minutes. During the test, the exhaled air in each expiration was evaluated by the Viasys metabolic measurement analyzer with the breath by breath method and automatically recorded to the computer. The criteria for reaching maximal exercise intensity were that the athlete could not continue the test, reaching the maximal heart rate, and falling the pedal speed below $60 \mathrm{Rpm}$. The test was ended in the presence of two of the three criteria. Oxygen consumption and carbon dioxide production graphs against time were continuously monitored during the test. The test was ended when the athlete showed dizziness, chest pain, severe dyspnea, pallor, bruising, confusion, and paroxysmal supraventricular tachycardia, prominent left bundle branch blocks, and a horizontal depression of more than $0.2 \mathrm{~mm}$ in the ST segment on the EKG. The test acceptance criteria were considered to be that reaching the $95 \%$ of the athlete's maximal heart rate (220-age) or the Respiratuar Quotient (RQ) value was above 1.0.

In our study, anaerobic capacity was measured by using the Wingate anaerobic performance test. The personal, physical characteristics and the contact information of the athletes were recorded. The seat height of the bicycle ergometer was adjusted to create a flexion angle of 15-200 at the athlete's knee joint, and a weight of 75 gram per kilogram of body was loaded onto the weight pan of the ergometer (Figure-2). First, the participants were asked to pedal the bicycle with all their strength without any weight loading. The maximum number of revolutions was noted. After a two-minute rest interval, the test was started. During the test, when the maximum speed was reached, the weight on the pan was reflected on the wheel as resistance. Athletes were asked to pedal against this resistance for 30 seconds using all their strength. As a result of the test, the maximum, average, and minimum power were automatically determined and recorded by the software of WAnT.

Hematological Measures: Participants were required to visit our laboratory to provide a blood sample from the forearm vein. Blood samples were obtained 2-3 days before the intervention (IHI) and 7-10 days post-intervention . Blood was analyzed for hemoglobin $(\mathrm{Hb})$, ferritin, erythrocytes and reticulocytes.

\section{Statistical analysis}

Statistical analysis was performed using the Statistical Package for the Social Sciences software version 17.0 (SPSS Inc., Chicago, IL, USA). Mean, median, standard deviation, minimum and maximum values were used to define the data. Evaluation of the data was done non-parametrically. A twoway repeated-measures ANOVA was performed to determine the effect of different treatments (IHI or NORMOXI) over time (pre/post) on all measured variables. 
In the comparisons between groups, Student-T test was used for data with normal distribution, and Mann-Whitney-U test was used for data that did not. Student-T (Paired samples T test) was used for dependent groups for within-group comparisons. Pearson correlation test was used to evaluate the relationships between variables and a $\mathrm{p}<0.05$ value was considered significant in statistical evaluations.

\section{RESULTS}

\section{Physical characteristics of athletes}

The ages, heights and weights of the athletes in the hypoxia and normoxia groups are given in Table 1, respectively. There was no statistically important difference between hypoxia and normoxia groups in terms of physical characteristics ( $\mathrm{p}>0.05)$.

Hematological parameters of athletes before/after intermittent hypoxic intervention

The hematological parameters of the athletes before and after the intermittent hypoxic intervention(IHI) were given in Table 2. No statistical difference was observed in the hypoxic and normoxia groups before and after hypoxic intervention $(\mathrm{p}>0.05)$

Aerobic performance values of the athletes before/after intermittent hypoxic intervention

The maximal oxygen consumption of the hypoxia group and the oxygen consumption values at the anaerobic threshold were given in Table 3. There was no statistical difference between $\mathrm{VO}_{2}$ max values and $\mathrm{VO}_{2}$ max values at anaerobic threshold before and after hypoxic intervention in both groups $(\mathrm{p}>0.05)$.
Anaerobic performance values of the cases before/after intermittent hypoxic intervention

Anaerobic power performance parameters of both groups were given in Table 4. In the hypoxia group in which we investigated the effect of hypoxic intervention, the statistically important difference in anaerobic parameters was not found before and after intervention ( $\mathrm{p}>0.05)$.

\section{DISCUSSION}

Numerous conflicting studies have been reported on the effect of acute and chronic hypoxia on aerobic performance (11-14). Results of several studies for intermittent hypoxic intervention (IHI), shown that there was no increase in maximal oxygen consumption after intervention $(11,14)$. On the other hand, Bonetti et al reported that IHI increases the aerobic performance, but this was not associated with maximal oxygen consumption $(12,13)$. Gore et al. discussed that besides hematological parameters, changes in a number of cardiac, respiratory mechanisms, molecular and lactate buffering capacity might be effective in the development of aerobic performance after hypoxic training (15). Therefore, it is seen that all hypoxic training and intervention protocols, including IHI, are not effective only via hematological parameters at increasing maximal oxygen consumption. In a similar study on skiers, an increase of $5-10 \%$ in repeated ski sprint performance was determined after 15 sessions of IHI (16). The reason for this increase may be the long hypoxic interval exposure time and a performance test method known to the athletes as aerobic performance criterion.

Table 1. Physical characteristics of the athletes

\begin{tabular}{lccc} 
& IHI $(\mathbf{n}=\mathbf{2 0})$ & NORMOXI $(\mathbf{n}=\mathbf{2 0})$ \\
Age & Ortalama \pm SD $(\mathbf{M i n}-\mathbf{M a x})$ & Ortalama \pm SD $(\mathbf{M i n}-\mathbf{M a x})$ & $(P>0.05)$ \\
Weight & $24.8 \pm 2.87(21-30)$ & $25.46 \pm 2.09(21-29)$ \\
BMI $\left(\mathbf{k g} / \mathbf{m}^{\mathbf{2}}\right)$ & $67 \pm 3.67(58-72)$ & $64.4 \pm 3.08(58-70)$ \\
\hline
\end{tabular}

Table 2: Baseline and post-intervention hematological parameters of athletes

\begin{tabular}{lcccc} 
& & IHI & \multicolumn{2}{c}{ NORMOXIA } \\
& Baseline & After IHI & Baseline & Post Intervention \\
Hemoglobin $(\mathrm{gr} / \mathrm{dl})$ & $15.64 \pm 0.44$ & $15.72 \pm 0.43$ & $15.76 \pm 0.46$ & $15.78 \pm 0.45$ \\
Hematocrit & $46.53 \pm 1.33$ & $46.85 \pm 1.27$ & $45.34 \pm 1.52$ & $46.44 \pm 1.54$ \\
${\text { Eritrosit } / \mathrm{mm}^{3}}^{5.15 \pm 0.22}$ & $5.21 \pm 0.19$ & $5.11 \pm 0.17$ & $5.16 \pm 0.15$ \\
\hline
\end{tabular}

Table 3: Maximal oxygen and maximal oxygen consumption at anaerobic threshold test values of the groups at baseline and post-intervention

\begin{tabular}{lcccc} 
& \multicolumn{2}{c}{ IHI } & \multicolumn{2}{c}{ NORMOXIA } \\
& Baseline & Post IHI & Baseline & Post-intervention \\
$\mathrm{VO}_{2} \max (\mathrm{ml} / \mathrm{kg} / \mathrm{dk})$ & $66.12 \pm 1.58$ & $67.02 \pm 1.67$ & $67.76 \pm 1.32$ & $67.94 \pm 1.16$ \\
$\mathrm{VO}_{2} \max (\mathrm{ml} / \mathrm{kg} / \mathrm{dk})$ (Anaerobic threshold) & $57.78 \pm 1.41$ & $58.66 \pm 1.13$ & $59.91 \pm 2.16$ & $60.71 \pm 0.91$ \\
\hline
\end{tabular}

IHI: Intermittant Hypoxic Intervention, VO2 max: Maximal oxygen consumption, VO2 max (ml/kg/dk) (Anaerobik Threshold ): Oxygen consumption at anaerobic threshold

Table 4: Anaerobic performance values of the groups before and after the intervention

\begin{tabular}{ccccc} 
& \multicolumn{2}{c}{ IHI } & \multicolumn{2}{c}{ NORMOXIA } \\
& Baseline & Post IHI & Baseline & Post intervention \\
\hline Maximal Power watt/kg & $10.57 \pm 0.81$ & $10.68 \pm 0.79$ & $11.47 \pm 0.87$ & $11.44 \pm 0.80$ \\
Mean Power watt/kg & $6.13 \pm 0.34$ & $6.16 \pm 0.42$ & $6.08 \pm 0.23$ & $6.16 \pm 0.28$ \\
Minimum Power watt/kg & $3.10 \pm 0.37$ & $3.17 \pm 0.49$ & $3.31 \pm 0.41$ & $3.27 \pm 0.53$ \\
\hline
\end{tabular}


Babrock et al. also did not find a significant change as a result of 19 sessions of IHI ( $5 \mathrm{~min}$ hypoxia, $5 \mathrm{~min}$ normoxia) intervened by 10 recreational skiers for 3 weeks, based on lactate threshold and maximal oxygen consumption as aerobic performance criteria (17). In a study, a significant increase was found between the $3 \mathrm{~km}$ time-tested running tests performed on the 2 nd and 17th days after 17 sessions of 90 minute IHI ( 5 min hypoxia, $13-10 \% \mathrm{FiO}_{2} 5 \mathrm{~min}$ normoxia) to 22 non-elite athletes in different athletics branches (18). This increase may be due to lower hypoxia dose, longer application sessions and differences in the training level of the athletes. Hinckson et al. also did not detect any significant change in $5 \mathrm{~km}$ running performance against time after 20 sessions of 90 minutes of IHI (6 minutes hypoxia and 4 minutes normoxia; $\mathrm{SpO}_{2} 92-80 \%$ ) on 11 elite mountaineers, seven women and four men (19).

It is accepted that hypoxia does not have a positive effect on anaerobic performance. In our study, we did not find a significant increase in the average and maximal power of elite distance runners in the Wingate test. Tadibi et al. did not report an increase in average and maximal strength in endurance athletes after 15 sessions of IHI, evaluated with the Wingate test (11). Likewise, Bonetti et al. did not detect a significant increase in repetitive sprint times in elite cyclists and triathletes after 15 sessions of IHI (10). Bonetti et al. again did not show an increase in peak and mean strength in the elite skiers with a control group of 10, after 19 sessions of 4 weeks of IHI (13). In another study, Bonetti et al. found no difference in repetitive sprint times in 18 elite triathletes without a control group with a similar IHI protocol as in the previous study (12). Also, Hinckson et al. did not find a difference in repeated sprint performance tests against time after 14 sessions of IHI (6 min hypoxia, 4 min normoxia ) in 10 rugby players (20). As an anaerobic performance test, studies were mostly evaluated with sport-specific tests such as repeated sprint tests against time that the athletes were familiar with. With the Wingate test we used, Tadibi et al. did not report an increase in mean and maximal strength after 15 sessions of IHI (6 min hypoxia $10-11 \% \quad \mathrm{FiO}_{2}, 4 \mathrm{~min}$ normoxia) at ten marathoners with the control group (10).

The purpose of hypoxia is to increase the release of Erythropoietin (EPO) hormone by accelerating the synthesis of erythrocyte precursor series in the bone marrow and; as a result to improve the oxygen carrying capacity to the tissues (21, 23). In our study, also an increase in hemoglobin, hematocrit and erythrocyte levels were not observed as a result of a study performed with 15 sessions of IHI $(6 \mathrm{~min}$ hypoxia, 4 min normoxia; $10-11 \% \mathrm{FiO}_{2}$ ) to the elite athlete hypoxia group of 20 distance runners (11). After 20 sessions of IHI (5 min hypoxia, 5 min normoxia; $10-12 \% \quad \mathrm{FiO}_{2}$ ) performed on 14 elite skiers with the control group, in which EPO was studied, no difference was observed in the amount of erythrocytes and hemoglobin together with the amount of EPO hormone (14). After 15 sessions of IHI (5 min hypoxia, 5 min normoxia; $\mathrm{SpO}_{2} 90-76 \%$ ) at elite skiers and cyclists, no significant change was observed in the amounts of reticulocyte, hemoglobin, and 2-3 diphosphoglycerate (12, 13). In another study, Hinckson et al did not report a significant change in hemoglobin, hematocrit and reticulocyte measurements after two days of 14 sessions of IHI (6 min hypoxia, 4 min normoxia; $\mathrm{SpO}_{2} 100-76 \%$ ) in 10 elite rugby players (20). Although increased physical performance increases with IHI were observed in some studies, it was evaluated that these increases were not related to hematological parameters (24). Therefore, when people living at high altitudes in 38 different parts of the world were examined, it was found that there were significant hematological differences compared to each other (24). While the inhabitants of the Andes mountain were significantly different from those living at sea level, this ratio was found to be relatively normal for the inhabitants of Tibet (24).

We evaluated that a 4 week IHI program did not contribute to the aerobic and anaerobic performance of elite distance athletes. We did not observe the expected increases in hematological parameters, which is the most emphasized mechanism in terms of performance improvement.

\section{CONCLUSION}

Contrary to the studies in the literature with acute and chronic hypoxic intervention, we found that intermittent hypoxic intervention did not provide aerobic and anaerobic performance enhancement. There was no definite consensus on what the dose and duration of the hypoxic interval should be in previous studies. Aerobic and anaerobic performance enhancement can be achieved by increasing the dose or the duration of the hypoxic intervention. It may be another research topic that IHI can provide performance increase in sub-elite or recreational athletes instead of elite athletes applied in our study.

\section{Acknowledgments: None}

Author Contributions: AE, TA: Data collection, Formal analysis, Methodology, Project administration, Statistical Analyses, AE: Article writing and revisions

Financial \& competing interest's disclosure: The authors have no relevant affiliations or financial involvement with any organisation or entity with a financial interest in or financial conflict with the subject matter or materials discussed in the manuscript. This includes employment, consultancies, honoraria, stock ownership or options, expert testimony, grants or patents received or pending, or royalties.

Conflict of interest: The authors declared no potential conflicts of interest with respect to the research, authorship, and/or publication of this article. This research did not receive and specific grant from funding agencies in the public, commercial or not-for-profit sectors.

\section{REFERENCES}

1. Easton PA, Slykerman LJ, Anthonisen NR. Ventilatory response to sustained hypoxia in normal adults. J Appl Physiol 1986; 61(3),906-11.

2. Klausen T, Christensen H, Hansen J, Nielsen O, Fogh-Andersen N, Olsen. Human erythropoietin response to hypocapnic hypoxia, normocapnic hypoxia and hypocapnic normoxia. Eur J Appl Physiol 1996; 74,475-80.

3. Peltonen JE, Tikkanen HO, Rusko HK. Cardiorespiratory responses to exercise in acute hypoxia, hyperoxia and normoxia. Eur J Appl Physiol 2001;85,(1):82-8.

4. Powell FL, Milsom WK, Mitchell GS. Time domains of the hypoxic ventilatory response. Respir Physiol 1998; 112(2),123-34. 
5. Vogel JA, Harris CW. Cardiopulmonary responses of resting man during early exposure to high altitude. J Appl Physiol $1967 ; 22(6), 1124-8$.

6. Wagner PD, Gale GE, Moon RE, Torre-Bueno JR, Stolp BW, Saltzman HA. Pulmonary gas exchange in humans exercising at sea level and simulated altitude. J Appl Physiol 1986;61(1),260-70.

7. Dwindell MR, Janssen PL, Bisgard GE. Lack of long term facilitation of ventilation after exposure to hypoxia in goats. Respir Physiol $1997 ; 108(1), 1-9$

8. Powell FL, Garcia N. Physiological effects of intermittent hypoxia High Altitude Medicine and Biology 2000; 1(2),125-36

9. Bonetti DL, Hopkins WG, Kilding AE. High-intensity kayak performance after adaptation to intermittent hypoxia. Int J Sports Physiol Perform 2006;(1),246-60.

10. Bonetti DL, Hopkins WG. Sea-level exercise performance following adaptation to hypoxia: A meta-analysis. Sports Med 2009;39(2),107-27.

11. Tadibi V, Dehnert C, Menold E, Bartsch P. Unchanged anaerobic and aerobic performance after short-term intermittent hypoxia. Med Sci Sports Exerc 2007; 39(5), 858-64.

12. Bonetti DL, Hopkins WG, Lowe TE, Kilding AE. Cycling performance following adaptation to two protocols of acutely intermittent hypoxia. Int J Sports Physiol Perform 2009; 4,66-83.

13. Bonetti DL, Hopkins WG, Kilding AE. High-intensity kayak performance after adaptation to intermittent hypoxia. Int J Sports Physiol Perform 2006;. 1,246-60.

14. Julian CG, Gore CJ, Wilber RL, Daniels JT, Fredricson M, Stray Gundersen J. Intermittent normobaric hypoxia does not alter performance or erythropoietic markers in highly trained distance runners. J Appl Physiol 2004; 96,1800-07.

15. Gore CJ, Clark SA, Saunders PU. Nonhaematological mechanisms of improved sea-level performance after hypoxic exposure. Med Sci Sports Exerc 2007;39(9),1600-9.
16. Bonetti DL, Hopkins WG, Kilding AE. High-intensity kayak performance after adaptation to intermittent hypoxia. Int J Sports Physiol Perform 2006; 1,246-60.

17. Babcock CJ, Kirby TE. The effect of intermittent simulated altitude exposure via re-breathing on cycling performance. Med Sci Sports Exerc 2008; 40(5),51.

18. Hamlin MJ, Hellemans J. Effect of intermittent normobaric hypoxic exposure at rest on haematological, physiological, and performance parameters in multi-sport athletes. J Sports Sci 2007;25(4),431-41.

19. Hinckson EA, Hopkins WG, Downey BM, Smith TBRJ. The effect of intermittent hypoxic training via a hypoxic inhaler on physiological and performance measures in rowers: A pilot study. J Sci Med Sport.2006; $9(1), 177-80$.

20. Hinckson EA, Hamlin MJ, Wood MR, Hopkins WG(2007). Game performance and intermittent hypoxic training. $\mathrm{Br} \mathrm{J}$ Sports Med. 41(8),537-9.

21. Levine BD, Stray-Gundersen J. Point: Positive effects of intermittent hypoxia (live high: train low) on exercise performance are mediated primarily by augmented red cell volume. J Appl Physiol 2005;99,2053-

22. Levine BD, Stray-Gundersen J, Gore CJ, Hopkins WG. "Living hightraining low": effect of moderate-altitude acclimatization with lowaltitude training on performance. J Appl Physiol 1997; 83,102-12.

23. Stray-Gundersen J, Chapman RF, Levine BD(1997). "Living hightraining low" altitude training improves sea level performance in male and female elite runners. J Appl Physiol 1997; 91(3):1113-20.

24. Akgün N. Exercise phsiology. 1992;4.Bask1 GSGM yayınları, Ege Üniversitesi Basımevi İzmir 